Network Working Group

Request for Comments: 750

NIC: 45500

Obsoletes: 739,

$604,503,433,349$
J. Postel

USC-ISI

26 September 1978

\title{
ASSIGNED NUMBERS
}

This Network Working Group Request for Comments documents the currently assigned values from several series of numbers used in network protocol implementations. This RFC will be updated periodically, and in any case current information can be obtained from Jon Postel. The assignment of numbers is also handled by Jon. If you are developing a protocol or application that will require the use of a link, socket, etc. please contact Jon to receive a number assignment.

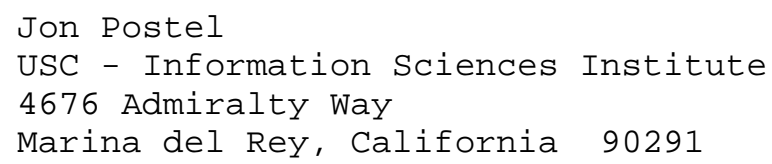

Most of the protocols mentioned here are documented in the RFC series of notes. The more prominent and more generally used are documented in the Protocol Handbook [1] prepared by the Network Information Center (NIC). In the lists that follow a bracketed number, e.g. [1], off to the right of the page indicates a reference for the listed protocol. 
NWG/RFC 750

Assigned Numbers

Assigned Link Numbers

\section{ASSIGNED LINK NUMBERS}

The word "link" here refers to a field in the original ARPANET Host/IMP interface leader. The link was originally defined as an 8 bit field. Some time after the ARPANET Host-to-Host (AHHP) protocol was defined and, by now, some time ago the definition of this field was changed to "Message-ID" and the length to 12 bits. The name link now refers to the high order 8 bits of this 12 bit message-id field. The low order 4 bits of the message-id field are to be zero unless specifically specified otherwise for the particular protocol used on that link. The Host/IMP interface is defined in BBN report 1822 [2].

Link Assignments:

\begin{tabular}{|c|c|c|c|}
\hline Decimal & Octal & Description & References \\
\hline------- & ----- & ------------ & ---------- \\
\hline 0 & 0 & AHHP Control Messages & {$[1,3]$} \\
\hline 1 & 1 & Reserved & \\
\hline $2-71$ & $2-107$ & AHHP Regular Messages & {$[1,3]$} \\
\hline $72-151$ & $110-227$ & Reserved & \\
\hline 152 & 230 & PARC Universal Protocol & \\
\hline 153 & 231 & TIP Status Reporting & \\
\hline 154 & 232 & TIP Accounting & \\
\hline $155-158$ & $233-236$ & Internet Protocol & {$[35,36,42,43,44]$} \\
\hline $159-191$ & $237-277$ & Measurements & [28] \\
\hline $192-195$ & $300-303$ & Message Switching Protocol & {$[4,5]$} \\
\hline $196-255$ & $304-377$ & Experimental Protocols & \\
\hline $224-255$ & $340-377$ & NVP & {$[1,39$} \\
\hline
\end{tabular}


NWG/RFC 750

Assigned Numbers

Assigned Socket Numbers

\section{ASSIGNED SOCKET NUMBERS}

Sockets are used in the AHHP [1,3] to name the ends of logical connections which carry long term conversations. For the purpose of providing services to all callers an Initial Connection Procedure ICP $[1,34]$ is used between the user process and the server process. This list specifies the socket used by the server process as its contact socket.

Socket Assignments:

General Assignments:

$\begin{array}{lll}\text { Decimal } & \text { Octal } & \text { Description } \\ ------ & ----- & --------- \\ 0-63 & 0-77 & \text { Network Wide Standard Function } \\ 64-127 & 100-177 & \text { Hosts Specific Functions } \\ 128-223 & 200-337 & \text { Reserved for Future Use } \\ 224-255 & 340-377 & \text { Any Experimental Function }\end{array}$

Specific Assignments:

Network Standard Functions

\begin{tabular}{|c|c|c|c|}
\hline Decimal & Octal & Description & References \\
\hline & & ----------- & \\
\hline 1 & 1 & Old Telnet & [6] \\
\hline 3 & 3 & Old File Transfer & {$[7,8,9]$} \\
\hline 5 & 5 & Remote Job Entry & {$[1,10]$} \\
\hline 7 & 7 & Echo & [ 11$]$ \\
\hline 9 & 11 & Discard & [12] \\
\hline 11 & 13 & Who is on or SYSTAT & \\
\hline 13 & 15 & Date and Time & \\
\hline 15 & 17 & Who is up or NETSTAT & \\
\hline 17 & 21 & Short Text Message & \\
\hline 19 & 23 & Character generator or TTYTST & [13] \\
\hline 21 & 25 & New File Transfer & {$[1,14,15]$} \\
\hline 23 & 27 & New Telnet & {$[1,16,17]$} \\
\hline 25 & 31 & Distributed Programming System & {$[18,19]$} \\
\hline 27 & 33 & NSW User System w/COMPASS FE & {$[20]$} \\
\hline 29 & 35 & $\mathrm{MSG}-3 \mathrm{ICP}$ & [21] \\
\hline 31 & 37 & MSG-3 Authentication & [21] \\
\hline 33 & 41 & DPS ICP & {$[18,19]$} \\
\hline 35 & 43 & IO Station Spooler & \\
\hline 37 & 45 & Time Server & {$[1,22$} \\
\hline 39 & 47 & NSW User System w/SRI FE & {$[20$} \\
\hline 41 & 51 & Graphics & {$[1,26$} \\
\hline $42-63$ & $52-77$ & unassigned & \\
\hline
\end{tabular}




\begin{tabular}{|c|c|c|c|}
\hline Decimal & Octal & Description & References \\
\hline------- & ----- & ----------- & ---------- \\
\hline 65 & 101 & Speech Data Base at LL-TX-2 & [23] \\
\hline 67 & 103 & Datacomputer at $\mathrm{CCA}$ & {$[24]$} \\
\hline 69 & 105 & CPYNET & \\
\hline 71 & 107 & NETRJS (EBCDIC) at UCLA-CCN & {$[1,25]$} \\
\hline 73 & 111 & NETRJS (ASCII-68) at UCLA-CCN & {$[1,25]$} \\
\hline 75 & 113 & NETRJS (ASCII-63) at UCLA-CCN & {$[1,25]$} \\
\hline 77 & 115 & any private RJE server & \\
\hline 79 & 117 & Name or Finger & {$[1,40]$} \\
\hline 81 & 121 & Network BSYS & \\
\hline 83 & 123 & MIT ML Device & \\
\hline 85 & 125 & MIT ML Device & \\
\hline $86-94$ & $126-136$ & unassigned & \\
\hline 95 & 137 & SUPDUP & [33] \\
\hline 97 & 141 & Datacomputer status & \\
\hline $98-127$ & $142-136$ & unassigned & \\
\hline Reserved & for Future & Use & \\
\hline Decimal & Octal & Description & References \\
\hline------- & ----- & ----------- & ---------- \\
\hline $128-223$ & $200-337$ & reserved & \\
\hline \multicolumn{4}{|c|}{ Experimental Functions } \\
\hline Decimal & Octal & Description & References \\
\hline------- & ----- & ------------ & ---------- \\
\hline $224-231$ & $340-347$ & unassigned & \\
\hline $232-237$ & $350-355$ & Authorized Mailer at BBN & \\
\hline 239 & 357 & unassigned & \\
\hline 241 & 361 & NCP Measurement & {$[27,28]$} \\
\hline 243 & 363 & Survey Measurement & {$[28,29,30]$} \\
\hline 245 & 365 & LINK & {$[31]$} \\
\hline 247 & 367 & TIPSRV & \\
\hline $249-255$ & $371-377$ & RSEXEC & {$[31,32]$} \\
\hline
\end{tabular}


NWG/RFC 750

Assigned Numbers

Assigned Network Numbers

\section{ASSIGNED NETWORK NUMBERS}

This list of network numbers is used in the internetwork protocols now under development, the field is 8 bits in size.

Assigned Network Numbers

\begin{tabular}{|c|c|c|c|}
\hline Decimal & Octal & Network & References \\
\hline------- & ----- & ------- & ----------- \\
\hline 0 & 0 & Reserved & \\
\hline 1 & 1 & BBN Packet Radio Network & \\
\hline 2 & 2 & SF Bay Area Packet Radio Network (1) & \\
\hline 3 & 3 & BBN RCC Network & \\
\hline 4 & 4 & Atlantic Satellite Network & \\
\hline 5 & 5 & Washington D.C. Packet Radio Network & \\
\hline 6 & 6 & SF Bay Area Packet Radio Network (2) & \\
\hline 7 & 7 & CHAOS Network & \\
\hline 8 & 10 & BBN SATNET Test Network & \\
\hline 9 & 11 & Ft. Gordon Packet Radio Network & \\
\hline 10 & 12 & ARPANET & {$[1,2]$} \\
\hline 11 & 13 & University College London Network & \\
\hline 12 & 14 & CYCLADES & \\
\hline 13 & 15 & National Physical Laboratory & \\
\hline 14 & 16 & TELENET & \\
\hline 15 & 17 & British Post Office EPSS & \\
\hline 16 & 20 & DATAPAC & \\
\hline 17 & 21 & TRANSPAC & \\
\hline 18 & 22 & LCS Network & {$[37,38]$} \\
\hline 19 & 23 & TYMNET & \\
\hline 20 & 24 & Ft. Sill Packet Radio Network & \\
\hline 21 & 25 & DCEC EDN & \\
\hline $22-254$ & $26-376$ & Unassigned & \\
\hline 255 & 377 & Reserved & \\
\hline
\end{tabular}


NWG/RFC 750

Assigned Numbers

Assigned Internet Message Versions

\section{ASSIGNED INTERNET MESSAGE VERSIONS}

In the internetwork protocols there is a field to identify the version of the internetwork general protocol. This field is 4 bits in size.

Assigned Internet Message Versions

$\begin{array}{cc}\text { Decimal } & \text { Octal } \\ ------ & ----- \\ 0 & 0 \\ 1 & 1 \\ 2 & 2 \\ 3 & 3 \\ 4 & 4 \\ 5-14 & 5-16 \\ 15 & 17\end{array}$

Version
------
March 1977 version
January 1978 version
February 1978 version A
February 1978 version B
September 1978 version 4
Unassigned
Reserved

References

$[36]$

[42]

[43]

[44] 
NWG/RFC 750

Assigned Numbers

Assigned Internet Message Formats

\section{ASSIGNED INTERNET MESSAGE FORMATS}

In several of the internetwork protocol versions there is a field to identify the format of the host level specific protocol. This field is 8 bits in size. This field is called either Format or Protocol.

Assigned Internet Message Formats

$\begin{array}{rc}\text { Decimal } & \text { Octal } \\ ----- & ---- \\ 0 & 0 \\ 1 & 1 \\ 2 & 2 \\ 3 & 3 \\ 4 & 4 \\ 5 & 5 \\ 6 & 6 \\ 7-254 & 7-376 \\ 255 & 377\end{array}$

Format

References

Reserved

raw internet

[42]

$\mathrm{TCP}-3$

DSP

[36]

Gateway Monitoring Message

$[37,38]$

TCP-3. 1

[41]

TCP -4

[45]

Unassigned

[46]

[Page 7] 
NWG/RFC 750

Assigned Numbers

Assigned Internet Message Types

\section{ASSIGNED INTERNET MESSAGE TYPES}

In the March 1977 internetwork protocol [35] there is a field to identify the type of the message. This field is 4 bits in size.

Assigned Internet Message Types

$\begin{array}{rclr}\text { Decimal } & \text { Octal } & \text { Type } & \text { References } \\ ----- & ---- & --- & ------- \\ 1 & 0 & \text { Raw Internet Packet } & \text { [35] } \\ 2 & 1 & \text { TCP-2 } & \\ 3 & 2 & \text { Secure } & \\ 4 & 3 & \text { Gateway } & \text { [37 38] } \\ 5 & 5 & \text { Measurement } & \\ 6 & 6 & \text { DSP } & \\ 7-12 & 7-14 & \text { UCL } & \\ 13 & 15 & \text { Reserved } & \\ 14 & 16 & \text { Pluribus } & \\ 15 & 17 & \text { Telenet } & \end{array}$


NWG/RFC 750

Assigned Numbers

References

\section{REFERENCES}

[1] Feinler, E. "ARPANET Protocol Handbook," NIC 7104, Defense Communications Agency, January 1978.

[2] BBN, "Specifications for the Interconnection of a Host and an IMP," Report 1822, Bolt Beranek and Newman, Cambridge, Massachusetts, January 1976.

[3] McKenzie, A. "Host/Host Protocol for the ARPA Network," NIC 8246, January 1972. Also in [1].

[4] Walden, D. " A System for Interprocess Communication in a Resource Sharing Network," RFC 62, NIC 4962, 3 August 1970. Also published in Communications of the ACM, volume 15, number 4, April 1972 .

[5] Bressler, B. "A Proposed Experiment with a Message Switching Protocol," RFC 333, NIC 9926, 15 May 72.

[6] Postel, J. "Telnet Protocol," RFC 318, NIC 9348, 3 April 1972.

[7] McKenzie, A. "File Transfer Protocol," RFC 454, NIC 14333, 16 February 1973.

[8] Clements, R. "FTPSRV -- Extensions for Tenex Paged Files," RFC 683, NIC 32251, 3 April 1975. Also in [1].

[9] Harvey, B. "One More Try on the FTP," RFC 691, NIC 32700, 6 June 1975 .

[10] Bressler, B. "Remote Job Entry Protocol," RFC 407, NIC 12112, 16 October 72. Also in [1].

[11] Postel, J. "Echo Process," RFC 347, NIC 10426, 30 May 1972.

[12] Postel, J. "Discard Process," RFC 348, NIC 10427, 30 May 1972 .

[13] Postel, J. "Character Generator Process," RFC 429, NIC 13281, 12 December 1972 .

[14] Neigus, N. "File Transfer Protocol," RFC 542, NIC 17759, 12 July 1973. Also in [1].

[15] Postel, J. "Revised FTP Reply Codes," RFC 640, NIC 30843, 5 June 1974. Also in [1]. 
NWG/RFC 750

Assigned Numbers

References

[16] McKenzie, A. "Telnet Protocol Specification," NIC 18639 , August 1973. Also in [1].

[17] McKenzie, A. "Telnet Option Specification," NIC 18640, August 1973. Also in [1].

[18] White, J. "A High Level Framework for Network-Based Resource Sharing," RFC 707, NIC 34263, 14 January 1976. Also in NCC Proceedings, AFIPS, June 1976.

[19] White, J. "Elements of a Distributed Programming System," RFC 708, NIC 34353, 28 January 1976.

[20] COMPASS. "Semi-Annual Technical Report," CADD-7603-0411, Massachusetts Computer Associates, 4 March 1976. Also as, "National Software Works, Status Report No. 1," RADC-TR-76-276, Volume 1, September 1976. And COMPASS. "Second Semi-Annual Report," CADD-7608-1611, Massachusetts Computer Associates, 16 August 1976.

[21] NSW Protocol Committee, "MSG: The Interprocess Communication Facility for the National Software Works," CADD-7612-2411, Massachusetts Computer Associates, BBN 3237, Bolt Beranek and Newman, Revised 24 December 1976.

[22] Harrenstien, K. "Time Server," RFC 738, NIC 42218, 31 October 1977. Also in [1].

[23] Armenti, A., D. Hall, and A. Stone. "Lincoln speech Data Facility," SUR Note 37, NIC 10917, 14 July 1972.

[24] CCA, "Datacomputer Version 1 User Manual," Computer Corporation of America, August 1975.

[25] Braden, R. "NETRJS Protocol," RFC 740, NIC 42423, 22 November 1977. Also in [1].

[26] Sproull, R, and E. Thomas. "A Networks Graphics Protocol," NIC 24308, 16 August 1974. Also in [1].

[27] Cerf, V., "NCP Statistics," RFC 388, NIC 11360, 23 August 1972 .

[28] Cerf, V., "Formation of a Network Measurement Group (NMG)," RFC 323, NIC 9630, 23 March 1972.

[29] Bhushan, A., "A Report on the Survey Project," RFC 530, NIC 17375, 22 June 1973. 
NWG/RFC 750

Assigned Numbers

References

[30] Cantor, D., "Storing Network Survey Data at the Datacomputer," RFC 565, NIC 18777, 28 August 1973.

[31] Bressler, R., "Inter-Entity Communication -- An Experiment," RFC 441, NIC 13773, 19 January 1973.

[32] Thomas, R. "A Resource Sharing Executive for the ARPANET," AFIPS Conference Proceedings, 42:155-163, NCC, 1973.

[33] Crispin, M. "SUPDUP Protocol," RFC 734, NIC 41953, 7 October 1977. Also in [1].

[34] Postel, J. "Official Initial Connection Protocol," NIC 7101, 11 June 1971. Also in [1].

[35] Cerf, V. "Specification of Internet Transmission Control Program -- TCP (version 2)," March 1977.

[36] Cerf, V. and J. Postel, "Specification of Internetwork Transmission Control Program -- TCP Version 3," USC-Information Sciences Institute, January 1978.

[37] Reed, D. "Protocols for the LCS Network," Local Network Note 3, Laboratory for Computer Science, MIT, 29 November 1976.

[38] Clark, D. "Revision of DSP Specification," Local Network Note 9, Laboratory for Computer Science, MIT, 17 June 1977.

[39] Cohen, D. "Specifications for the Network Voice Protocol (NVP)," NSC Note 68, 29 January 1976. Also as USC-Information Sciences Institute RR-75-39, March 1976, and as RFC 741, NIC 42444, 22 November 1977. Also in [1].

[40] Harrenstien, K. "Name/Finger," RFC 742, NIC 42758, 30 December 1977. Also in [1].

[41] Cole, J. "Gateway Monitoring Messages," BBN, 1 February 1978.

[42] Postel, J. "Draft Internetwork Protocol Specification -Version 2," USC-Information Sciences Institute, February 1978.

[43] Cerf, V. "A Proposed New Internet Header Format," Advanced Research Projects Agency, IEN 26, 14 February 1978.

[44] Postel, J. "Internetwork Protocol Specification -- Version 4," IEN-54, USC-Information Sciences Institute, September 1978. 
NWG/RFC 750

Assigned Numbers

References

[45] Cerf, V. "A Proposal for TCP Version 3.1 Header Format," Advanced Research Projects Agency, IEN 26, 14 February 1978.

[46] Postel, J. "Specification of Internetwork Transmission Control Protocol -- Version 4," IEN-55, USC-Information Sciences Institute, September 1978. 